\title{
AN OPTIMIZED VEHICLE PARKING MECHANISM USING ARTIFICIAL NEURAL NETWORK
}

\author{
Ruby Singh \\ Research Scholar, Department of Computer Science and Engineering, \\ Shobhit Institute of Engineering and Technology, Uttar Pradesh, India \\ Dr. Niraj Singhal \\ Professor, Department of Computer Science and Engineering, \\ Shobhit Institute of Engineering and Technology, Uttar Pradesh, India
}

\begin{abstract}
Our country has developed rapidly for decades with lot of commercial buildings and well contacted roads with a growing number of automobiles. The transportation industry has become the backbone of economy because of its widespread use in trade and commerce. Therefore, parking the vehicles has become a consideration. For parking the vehicles in a parking space, old parking system is still used which is maintained in an unplanned mode and without any discipline. Because of this, people usually park their cars wherever they want, which can be a mess because most people don't follow any discipline. There should be some mechanism to park the vehicles appropriately; therefore, this research has introduced an optimized vehicle parking mechanism with the concept of Artificial Bee Colony (ABC) and Artificial Neural Network (ANN) which helps to get rid of the chaos usually faced.
\end{abstract}

Keywords: ABC, ANN, Parking Efficiency, Parking Mechanism

Cite this Article: Ruby Singh and Dr. Niraj Singhal, an Optimized Vehicle Parking Mechanism Using Artificial Neural Network, International Journal of Computer Engineering and Technology, 10(01), 2019, pp. 102-109.

http://www.iaeme.com/IJCET/issues.asp?JTypeIJCET\&VType=10\&IType=1

\section{INTRODUCTION}

Finding unoccupied parking spaces is a general problem in most of the cities, particularly in popular and well-trapped places such as shopping centers, sports venues and other tourist attractions [1]. This condition has to turn out to be more severe, chiefly in peak hours, whether it is the holiday season, the sale of carnival or some other fiesta. This issue occurs in most cases because patrons come here through their own means of transportation, resulting in large amounts of transportation or competing for some available parking spaces [2]. The inadequate 
accessibility of vacant parking spaces repeatedly leads to traffic congestion and frustration of drivers looking for accessible parking spaces [3]. Though, this is one of the main issue that causes traffic congestion. Interestingly, research on driver's parking behaviour suggests that this does not appear to avoid most drivers from waiting in line at their preferred parking lot, even for a long time [4]. In addition to limiting the driver's ability to find vacant parking spaces, the requirement of well-organized and accommodating parking systems has also resulted in this problem [5]. In order to solve this issue, the intelligent parking system has been executed in a number of countries like in Europe, Britain and even Asia as early as in the 1970s [6]. Intelligent parking systems focus on solving the right parking management problems by taking advantage of advances in technology that would surely assist in the alleviation. This research has proposed an enhanced vehicle parking mechanism using Artificial Bee Colony (ABC) and Artificial Neural Network (ANN) [7].

The organization of paper in the way that after the introduction part, section 2 highlights the existing work. Section 3 is the proposed architecture of an enhanced vehicle parking mechanism. Section 4 depicts the results and discussion which follows the parameters for the conduction of results. At last, Section 5 defines the crux and the consequences of the research work.

\section{STATE-OF-THE-ART}

This section reviews the study related to automatic parking management that tries to establish empty/occupied parking lots. The availability of car parking prediction is a demanding matter being studied for smart cities [1]. And generally these days when different car parking systems have sensing infrastructure linked to cloud-based system, the services of car parking let the drivers systemize the transports arrival and the departure. These services are a general way for forecasting the vacant rate of the parking spaces. Though, there is a provision of the probability of booking a parking spot, few accepted prediction methods considers that vehicles arrive at the car lots follows the poisson distribution. Later, the prediction of their capacity is done by Markov Chain [2,3] but the efficiency of these techniques is less as of the parking spaces demand is dependent on varied factors like day time, week data and weather conditions as well that are not acknowledged by these mechanisms. The promotions of large scale car parking are because of the smart city projects in the urban areas. So that the practitioners and researchers can have access to rational car park data sets. The investigation of Smart Santander on-street parking data has depicted that the parking and the vacant periods of varied parking area has a Weibull distribution [4]. In [5], three varied Machine Learning methods are applied for the prediction of car park occupancy rate for two data sets from San Francisco and Melbourne; supported vector machines, neural network and a regression tree, a neural network for investigating the relative weakness and the strength. SFpark was utilized as a use case for contrasting different spatio temporal clustering methods [6].

\section{PROBLEM STATEMENT}

Vehicle parking has become a significant issue these days and regularly the inevitability is growing. Mostly the manual vehicle parking system is followed and till now the problems such as time and fuel wastage, finding vacant space in the parking lot while parking the vehicle is increasing. Subsequent problem is the chaos that occurs while parking as there is no exact system for parking. Anyone usually park the vehicle anywhere that results in vehicle damage while coming in or moving out from the parking lot. Therefore, to resolve these issues we have introduced an efficient vehicle parking mechanism using Artificial Bee Colony and Artificial Neural Network. 


\section{PROPOSED ARCHITECTURE}

This section defines the proposed architecture of enhanced vehicle parking mechanism. Architecture is designed to prevent parking issues. The figure shows that a parking system is divided into three slots for a small car, SUV car, and bikes. A particular space has been provided to each particular car with width $\mathrm{W} 1, \mathrm{~W} 2$ and for bikes, space is given with width B0, B1, and B2. The architecture is shown below in fig 1 .

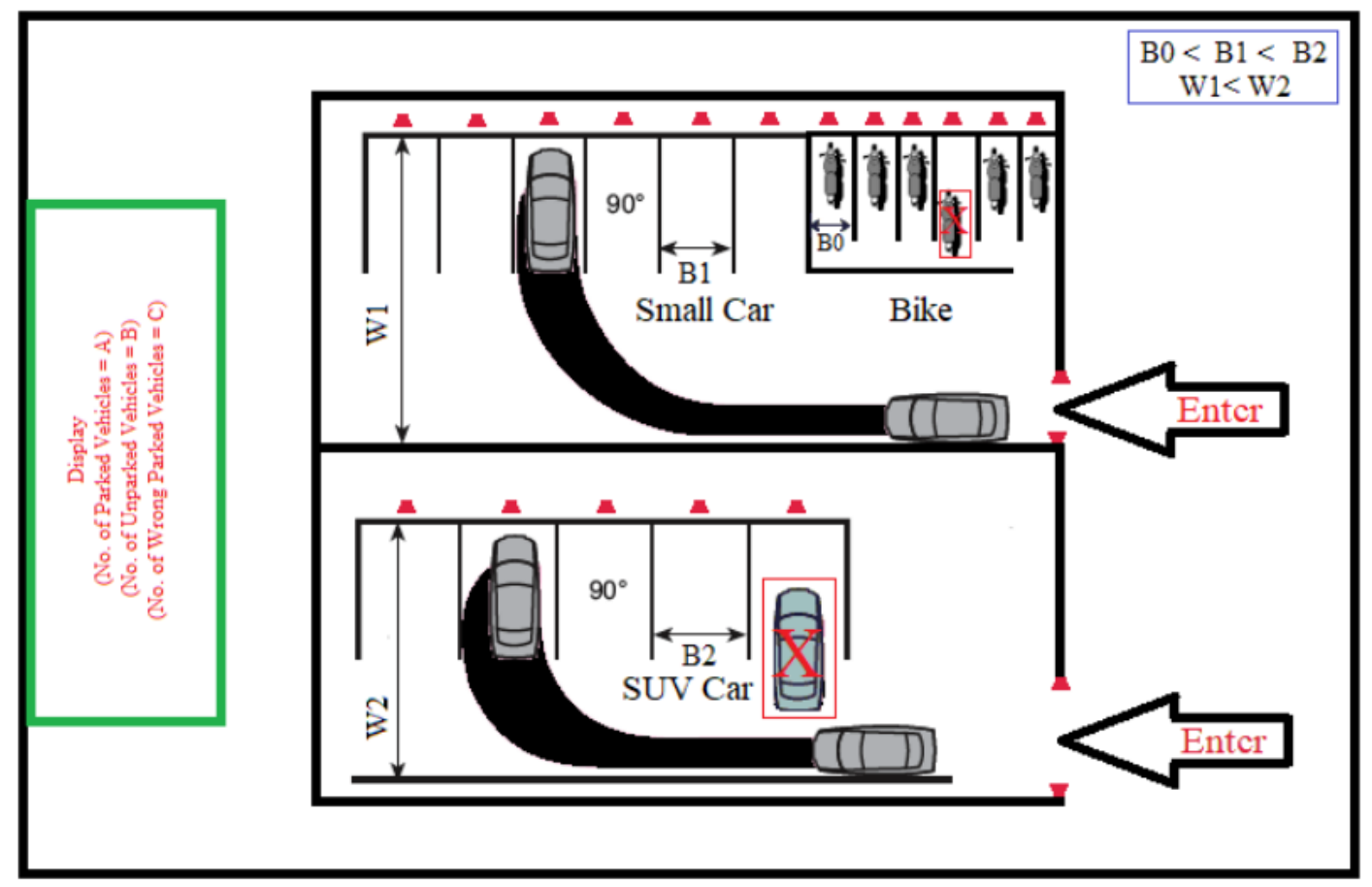

Figure 1: Enhanced Parking Architecture

$\mathrm{B} 0$ is the least provided space as depicted and the variation of the space increases with B1 and B2. There are two entry points from where vehicle can enter for parking in parking area. The entry points have been provided as per the size of vehicles (small, large) with sensors. The entry point sensors are used to count the number of vehicles entered in the parking lot and therefore, by this we can analyses the vacant space.

In every parking lot, there is a sensor which is used to find the position of vehicle. The driver should park the car by maintaining a distance with the sensors so that sensors can depict the exact position of the vehicle. If the position and distance of the vehicle are not satisfactory then the sensor will buzz an alarm to indicate the wrong manner of parking.

To check the position of a vehicle parked in the parking lot, the sensor will calculate the distance from sensor points. Let us suppose the co-ordinate of sensor is $\left(\mathrm{x}_{1}, \mathrm{y}_{1}\right)$ and co-ordinate of the vehicle is $\left(\mathrm{x}_{2}, \mathrm{y}_{2}\right)$. The sensor will calculate the exact distance of vehicles from sensor position using below equation:

$$
\text { Vehicle distance }=\sqrt{\left(x_{2}-x_{1}\right)^{2}+\left(y_{2}-y_{1}\right)^{2}}
$$

The above expression calculates the distance of vehicle from sensor's position. The vehicle should be parked within the appropriate range being marked. If any of the driver could not park the vehicle within the range then alarm will be buzzed and that very vehicle will come under the category of wrongly parked vehicles on the display board. While parking the vehicle, it should be kept in mind that the vehicle should be of that bandwidth as per the vehicle size. Therefore, in this research paper an optimized vehicle parking mechanism is proposed based on the concept of Artificial intelligence. In this work, Artificial Neural Network (ANN) is used 
as an Artificial Intelligence technique to maintain the parking system along with the Artificial Bee Colony $(\mathrm{ABC})$ optimization algorithm. The proposed $\mathrm{ABC}$ and $\mathrm{ANN}$ algorithms are given below:

\section{Algorithm 1: ABC Algorithm}

Input: Properties of parking space and Fitness Function

Output: Optimized properties of parking space

1 Define: Fit Fun - Fitness Function: Fit Fun $=\left\{\begin{array}{lr}\text { Ture } ; & \text { if } P_{\text {Val }}<\text { Threshold }_{\text {Val }} \\ \text { False } ; & \text { Otherwise }\end{array}\right.$

Where, $P_{V a l}:$ are properties of parking slots which are used for the parking purpose and Threshold $_{\text {Val }}$ is the threshold value of properties which define on the basis of the mean of all types parking space of properties.

2 Calculate, $\mathrm{R}$ as row and $\mathrm{C}$ as columns of properties array (Prop)

3 Initialize ABC parameters - Iterations (T)

- Total Bee (B)

- Selection function $\left(P_{V a l}\right.$ and Threshold Val $)$

4 Optimized Prop $=[]$

5 for $\mathrm{i}=1 \rightarrow \mathrm{R}$

6 for $\mathrm{j}=1 \rightarrow \mathrm{C}$

$7 P_{\text {Val }}=\sum_{i=1}^{B} \operatorname{Prop}(\mathrm{i})=\mathrm{E}_{\text {Bee }}$

8 Threshold $_{\text {Val }}=\frac{\sum_{i=1}^{B} \text { Prop (i) }}{\text { Length of Prop }}=O_{\text {Bee }}$

9 Fit Fun $=$ Fit Fun $\left(P_{\text {Val }}\right.$ Threshold Val $\left.)\right)$

$10 \quad$ Optimized Prop $=A B C$ (Fit Fun, Initialize Parameters) $\mathbf{1 1}$ end

12 end

13 Returns: Optimized properties of parking space

14 end

$\mathrm{ABC}$ algorithm is used to optimize the property of parking lots based on the distance and area. Basically, this algorithm is used in improving the efficiency of proposed work based on the fitness function. $\mathrm{ABC}$ is a swarm intelligence technique which acts as a meta-heuristic algorithm to solve the complex problem of parking. So in the above algorithm, properties of parking space and Fitness Function are taken as an Input and on the basis of the fitness function, $\mathrm{ABC}$ algorithm returns an optimized property set for the parking system. The output of the ABC Algorithm acts as an input of ANN which helps to classify the appropriate parking lot and to find the wrongly parked vehicle. The ANN algorithm is given below:

\section{Algorithm 2: Artificial Neural Network} (N)

Input: Optimized properties of parking space (T), Types of parking space $(\mathrm{G})$ and Neurons

Output: Parking Space

Training:

\section{Initialize the basic parameters of ANN}

- Number of Epochs (E) // Iterations used by ANN

- Number of Neurons (N)

- Performance parameters of training: MSE 
-Techniques: Levenberg Marquardt

- Data Division: Random

2 for $\mathrm{i}=1 \rightarrow \mathrm{T}$

3 if T belongs to $1^{\text {st }}$ type's parking space array

4 Group (1) = Properties of training data according to the available parking space

5 else if T belongs to $2^{\text {nd }}$ type's parking space array

6 Group (2) = Properties of training data according to the unavailable parking space

7 else

8 Group (3) = Properties of training data according to the wrongly parked parking space

9 end

10 end

11 Initialized the ANN using Training data and Group

$12 \mathrm{Net}=\operatorname{Newff}(T, G, N)$

13 Set the training parameters according to the requirements and train the system

14 Net $=$ Train (Net, Training data, Group)

Testing:

15 Test data $=$ Properties of current parking space

16 Parking Space $=$ simulate $($ Net, Test data)

\section{7 if Parking Space is available}

18 Parking Space $=$ Right

19 else

20 Parking Space $=$ Wrong

21 end

22 Return; Parking Space as output

23 end

Above algorithm is for ANN in which three Inputs; namely, optimized properties of parking space $(\mathrm{T})$, Types of parking space $(\mathrm{G})$ and Neurons $(\mathrm{N})$. As a training data, the optimized property of parking space is provided as an input. According to this property, we have defined three targets of Neural Network (NN) that is available space, unavailable space and wrong parking space. On the basis of training data and target, $\mathrm{NN}$ is trained using neurons as a carrier which helps to carry out the information from one layer to another layer.

\section{RESULT AND DISCUSSION}

This section defines the result obtained after the simulation of proposed architecture. For the simulation, parking efficiency parameter is computed. Parking Efficiency computation is shown below in Table I. 
An Optimized Vehicle Parking Mechanism Using Artificial Neural Network

Table I: Parking Efficiency Computation

\begin{tabular}{|c|c|c|c|}
\hline Time (HH: MM) & Parked Vehicles & $\begin{array}{c}\text { Non-Parked Vehicles } \\
\text { (Without AI) }\end{array}$ & $\begin{array}{c}\text { Non-Parked Vehicles } \\
\text { (With AI) }\end{array}$ \\
\hline $08: 00$ & 290 & 70 & 17 \\
\hline $09: 00$ & 310 & 83 & 19 \\
\hline $10: 00$ & 430 & 81 & 15 \\
\hline $11: 00$ & 420 & 78 & 15 \\
\hline $12: 00$ & 440 & 89 & 18 \\
\hline $13: 00$ & 390 & 64 & 16 \\
\hline $14: 00$ & 250 & 57 & 15 \\
\hline $15: 00$ & 390 & 76 & 17 \\
\hline $16: 00$ & 410 & 88 & 16 \\
\hline $17: 00$ & 435 & 92 & 18 \\
\hline $18: 00$ & 450 & 96 & 19 \\
\hline $19: 00$ & 410 & 85 & 16 \\
\hline $20: 00$ & 360 & 68 & 19 \\
\hline
\end{tabular}

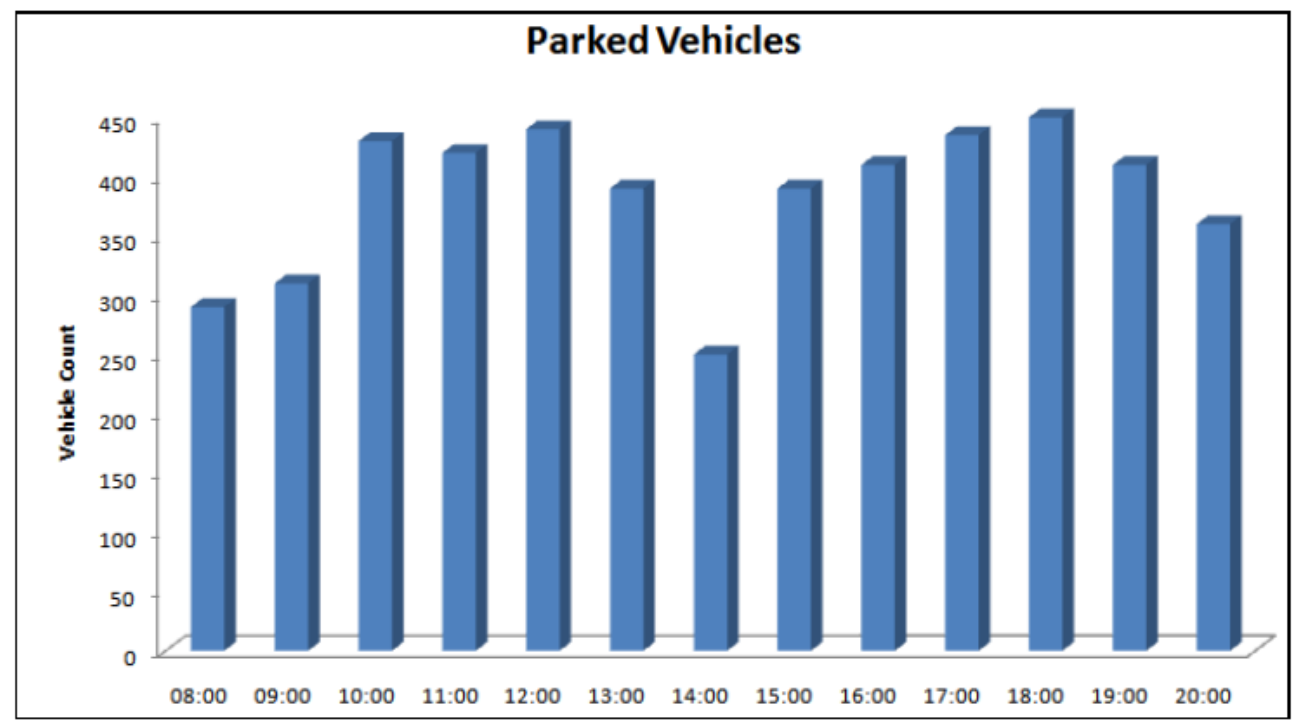

Figure 2: Number of Parked Vehicles

Fig 2 is the representation of number of parked vehicles from 08:00 to 20:00 hrs. It has been analyzed from the figure that the probability of parked vehicles increases from 10:00 to 12:00 and 16:00 to 18:00. For the analysis of proposed work, the capacity of 500 vehicles in a parking lot is considered. 


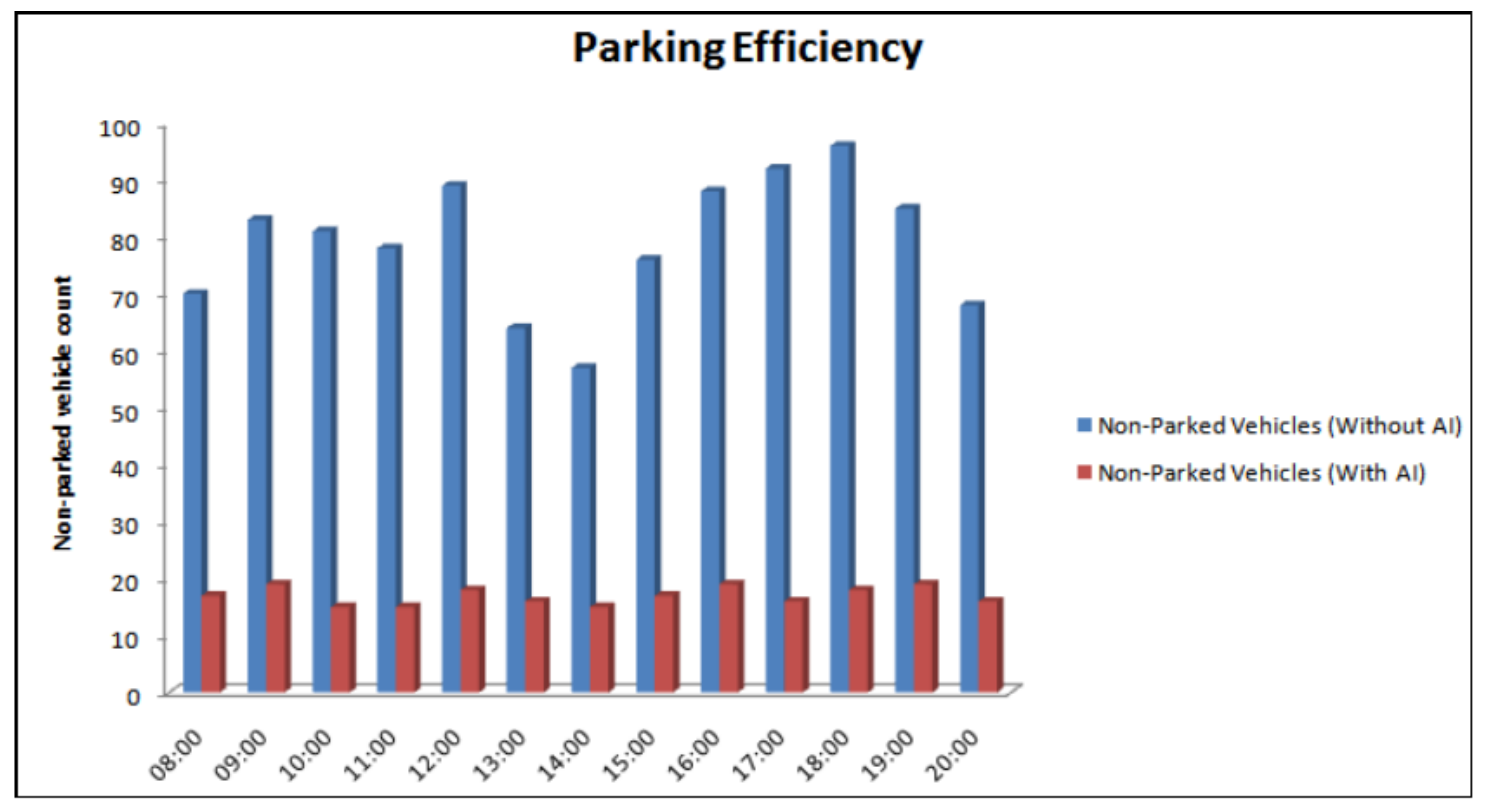

Figure 3: Parking Efficiency

Fig 3 represents the parking efficiency of the proposed optimized parking mechanism. The computation of parking efficiency is with respect to time. The examination of parking efficiency is from 08:00 to 20:00 hrs and the graph represents comparison when in the parking system, Artificial Intelligence (AI) is used and when AI is not used. From the observation, it is clear that when the concept of AI is used, the probability of wrong parking is less. It is because of the utilization of AI as the proposed work has used an alarming system when some vehicles are wrongly parked in the parking lot. The average of Non-Parked Vehicles (Without AI) is 79 whereas the average of Non-Parked Vehicles (With AI) is 16.92.

\section{CONCLUSION}

With the increase of population, the utilization of parking space is also amplified. The main problem being suffered is the erroneous utilization of the parking mechanism. If we talk generally, a large number of people follow the manual parking system with a number of turmoils. This issue has to be taken into consideration, so in this research, an optimized parking mechanism is proposed using Artificial Bee Colony (ABC) and Artificial Neural Network (ANN) which helps to minimize the factors that lead to the problems. To estimate the effectiveness of proposed work, parking efficiency parameter is considered. The average of Non-Parked Vehicles (Without AI) is 79 whereas the average of Non-Parked Vehicles (With AI) is 16.92 .

\section{REFERENCES}

[1] Lin T., Rivano H. and Mouel F.L, A Survey of Smart Parking solutions, IEEE Transactions on Intelligent Transportation Systems, 18(12), pp. 3229-3253, 2017.

[2] Klappenecker A., Lee H. and Welch J.L., Finding Available Parking Spaces made easy, Ad Hoc Networks, 12, pp. 243-249, 2014.

[3] Pullola S., Atrey P.K. and Saddik A.E., Towards an Intelligent GPS based Vehicle Navigation System for finding street parking lots, IEEE International Conference on Signal Processing and Communications, pp. 1251-1254, 2007. 
[4] Vlahogianni E.I., Kepaptsoglou K., Tsetsos V. and Karlaftis M.G., A Real Time Parking Prediction System for Smart Cities, Journal of Intelligent Transportation Systems, 20(2), pp.192-204, 2016.

[5] Zheng Y., Rajasegarar S. and Leckie C., Parking Availability Prediction for Sensor enabled Car Parks in smart cities, IEEE 10th International Conference on Intelligent Sensors, Sensor Networks and Information Processing (ISSNIP), pp. 1-6, 2015.

[6] Richter F., Martino S.D. and Mattfeld D.C., Temporal and Spatial clustering for a parking prediction service, IEEE $26^{\text {th }}$ International Conference on Tools with Artificial Intelligence, pp. 278-282, 2014.

[7] Rajabioun T., Foster B. and Ioannou P.A., Intelligent Parking Assistance, IEEE, Control and Automation (MED), 21st Mediterranean Conference, pp. 1156-1161, 2013.

[8] Rajabioun T. and Ioannou P.A., On-street and off-street Parking Availability Prediction using Multivariate spatio temporal models, IEEE Transactions on Intelligent Transportation Systems, 16(5), 2913-2924, 2015.

[9] Lin T., Smart Parking: Network Infrastructure and Urban Service, Ph.D. thesis, Lyon, INSA, 2015.

[10] Massobrio R. et al., Infrastructure Deployment in Vehicular Communication Networks using a parallel multi objective evolutionary algorithm, International Journal of Intelligent Systems, 32(8), 801-829, 2017.

[11] Morse G. and Stanley K.O., Simple Evolutionary Optimization can rival stochastic gradient descent in neural networks, Proceedings of the Genetic and Evolutionary Computation Conference, pp. 477-484, ACM, 2016.

[12] Reed R., et al., Similarities of Error Regularization, Sigmoid gain scaling, Target smoothing and training with jitter, IEEE Transactions on Neural Networks, 6(3), 529-538, 1995.

[13] Rumelhart D., Hinton G.E. and Williams R.j., Learning Internal Representations by Error Propagation, Technical Report No. ICS-8506, California University San Diego La Jolla Inst for Cognitive Science, 1985.

[14] Srivastava N., Hinton G., Krizhevsky A., Sutskever I. and Salakhutdinov R., Dropout: A Simple way to prevent Neural Networks from overfitting, Journal of Machine Learning Research, Springer, 2017.

[15] Stolfi D.H., Armas R., Alba E., Aguirre H. and Tanaka K., Fine Tuning of Traffic in our cities with Smart Panels: The Quito city case study, Proceedings of the Genetic and Evolutionary Computation Conference, pp. 1013-1019, ACM, 2016. 